Commun. math. Phys. 6, 49-60 (1967)

\title{
The Existence of Scalar Lie Fields*
}

\author{
JoHN H. LowensteIn \\ School of Physics and Astronomy \\ University of Minnesota \\ Received May 15, 1967
}

\begin{abstract}
It is shown that the existence of nontrivial scalar Lie fields (i. e. ficlds whose commutator is linear in the field itself) is not precluded by algebraic consistency arguments. A partial characterization of the simplest algebraic Lie field structures is given. Several examples are presented, one of which may be represented by Hermitian operators in a Hilbert space having a unitary representation of the Poincaré group.
\end{abstract}

\section{Introduction}

In discussing the general structure of relativistic quantum field theory it is often useful to have at one's disposal idealized models fulfilling some, though not all, of the usual field-theoretic postulates. One approach to the construction of such models, suggested by O. W. GREenBERG [1], is to consider the possibility of fields satisfying particularly simple commutation relations. A prime candidate is the so-called Lie field, for which the commutator is linear in the field itself [1] [2]. In the neutral scalar case this would mean

$$
i[A(x), A(y)]=\Delta(x, y)+\int d z c(x, y, z) A(z),
$$

where $\Delta$ and $c$ are real-valued generalized functions.

The usefulness of a nontrivial Lie field with an asymptotic particle interpretation (assuming such a model exists) is apparent, since the retarded functions can be calculated immediately once one knows the generalized functions $\Delta$ and $c$ (ŁopuszaNiskI [2] was the first to call attention to this aspect). Moreover, even without the asymptotic condition, a Lie field theory is soluble in the sense that the Wightman functions are uniquely determined by the specification of $\Delta$ and $c$ and the assumption of a positive energy spectrum [3].

The initial optimism regarding lie fields was dampened somewhat by a negative result of D. W. RobInson [4]. RobInson claimed that the existence of a scalar lie field which is nondegenerate, in the sense that $[[A(s), A(y)], A(z)]$ does not vanish identically, was precluded by in-

* Research supported by the National Science Foundation.

4 Commun.math. Phys., Vol. 6 
homogeneous Lorentz invariance and macroscopic locality alone. RoBInson's argument (which was not entirely valid, as we shall see in Section 3) depended critically on the number of invariants available, and so one could not conclude anything about the possibility of nonscalar Lie fields or of Lie fields over a space-time of dimension not equal to four. In particular, H. Lehmann (see [3]) gave a simple example for two-dimensional space-time (which could not be generalized to the fourdimensional case). The case against Lie fields was further strengthened by V. GLASER's result (see [3]) that a Lie field theory cannot be asymptotically complete.

In the present article (which is taken from part of the author's $\mathrm{Ph} . \mathrm{D}$. thesis [5]) it is shown that the pessimism regarding scalar Lie fields is not wholly warranted. Contrary to RoBINson's result, there exist many nondegenerate algebraic (scalar) Lie ficld structures consistent with Poincaré invariance and macroscopic locality. Whether or not any of these will be found useful in physics is a question which is left unanswered.

The author wishes to thank $\mathrm{R}$. HAAG for many valuable discussions.

\section{Algebraic Lie Field Structures}

Suppose that $A(x)$ is a real, scalar quantum field; i.e. the smeared out quantities

$$
A(f)=\int d^{4} x f(x) A(x)
$$

for appropriate real-valued test functions $f(x)$ over space-time are Hermitian operators in a Hilbert space. $A(x)$ is called a scalar Lie field if it satisfies equation (1.1), which may be rewritten in terms of smeared fields as

where

$$
i[A(f), A(g)]=\Delta(f, g)+A([f, g]),
$$

$$
\begin{aligned}
\Delta(f, g) & =\int d^{4} x d^{4} y f(x) g(y) \Delta(x, y) \\
{[f, g](z) } & =\int d^{4} x d^{4} y f(x) g(y) c(x, y, z) .
\end{aligned}
$$

It is convenient, as well as most natural from the mathematical standpoint, to consider first the algebraic properties of Lie fields without asking whether the quantities $A(f)$ have meaning as operators in a Hilbert space. The algebraic properties will be expressed wholly in terms of bilinear mappings on the test-function space (in other words, in terms of the generalized functions $\Delta$ and $c$ of equation (1.1)). We shall say that a test-function space ${ }^{1} \mathscr{K}$ has a Lie field structure if there exist continuous

1 The precise nature of $\mathscr{K}$ is irrelevant to the general algebraic discussion, but we shall assume that it contains the space of infinitely smooth functions of fast decrease. 
mappings

$$
\begin{aligned}
& (f, g) \in \mathscr{K} \times \mathscr{K} \rightarrow \Delta(f, g) \in \mathbb{R} \text { (real numbers) } \\
& (f, g) \in \mathscr{K} \times \mathscr{K} \rightarrow[f, g] \in \mathscr{K}
\end{aligned}
$$

such that for all $f, g, h \in \mathscr{K}$

(a) $[f, \alpha g+\beta h]=\alpha[f, g]+\beta[f, h]$

$$
\begin{aligned}
& {[f, \alpha g+\beta h]=\alpha[f, g]+\beta[f, h]} \\
& \Delta(f, \alpha g+\beta h)=\alpha \Delta(f, g)+\beta \Delta(f, h)
\end{aligned} \quad \text { all } \quad \alpha, \beta \in \mathbb{R}
$$

(b) $[f, g]=-[g, f]$

$$
\Delta(f, g)=-\Delta(g, f)
$$

(c) $[f,[g, h]]+[g,[h, f]]+[h,[f, g]]=0$

$\Delta(f,[g, h])+\Delta(g,[h, f])+\Delta(h,[f, g])=0$

(d) $\left[f_{L}, g_{L}\right]=[f, g]_{L}$

$$
\Delta\left(f_{L}, g_{L}\right)=\Delta(f, g)
$$

where $f_{L}(x)=f\left(L^{-1} x\right)$ for any inhomogeneous Lorentz transformation $L$.

(e) If, for any position-vector $x, T(x)$ is the translation by $x$, and if $a$ is an arbitrary fixed space-like vector, then for any $\varphi$ in $\mathscr{K}^{\prime}$, the space of continuous linear functionals on $\mathscr{K}$,

$$
\begin{aligned}
\lim _{\lambda \rightarrow \infty} \varphi\left(\left[f, g_{T(\lambda a)}\right]\right) & =0 \\
\lim _{\lambda \rightarrow \infty} \Delta\left(f, g_{T(\lambda a)}\right) & =0
\end{aligned}
$$

(f) $[\hat{f},[\hat{g}, \hat{h}]] \neq 0$ for some $\hat{f}, \hat{g}, \hat{h} \in \mathscr{K}$.

For some purposes one may wish to strengthen the macroscopic locality condition (e) by requiring that $\left[f, g_{T(\lambda a)}\right]$ tend to zero in the topology of $\mathscr{K}$; on the other hand, one may wish to weaken (e) by restricting the functionals $\varphi$ to some subspace of $\mathscr{K}^{\prime}$.

It is useful to rewrite some of the above algebraic properties in terms of the Fourier transforms $\tilde{\Delta}$ and $\tilde{c}$ of the generalized functions $\Delta$ and $c$ of equation (1.1). Because of Poincaré invariance and macroscopic locality, we may write

$$
\begin{aligned}
\tilde{c}(p, q, r) & =\delta(p+q+r) c^{\prime}(p, q) \\
\widetilde{\Delta}(p, q) & =\delta(p+q) \Delta^{\prime}(p),
\end{aligned}
$$

where $c^{\prime}$ and $\Delta^{\prime}$ depend only on the Lorentz invariants ${ }^{2}$. In addition we have

(i) $c^{\prime}(p, q)=-c^{\prime}(q, p)$

$$
\Delta^{\prime}(p)=-\Delta^{\prime}(-p)
$$

${ }^{2}$ Macroscopic locality forbids terms proportional to $\delta(p), \delta(q)$ or $\delta(r)$. 
(ii) $c^{\prime}(p, q) c^{\prime}(p+q, r)+c^{\prime}(q, r) c^{\prime}(q+r, p)+$

$$
\begin{gathered}
+c^{\prime}(r, p) c^{\prime}(r+p, q)=0 \\
c^{\prime}(p, q) \Delta^{\prime}(p+q)+c^{\prime}(-(p+q), p) \Delta^{\prime}(-q)+ \\
+c^{\prime}(q,-(p+q)) \Delta^{\prime}(-p)=0, \\
\text { (iii) } c^{\prime}(p, q)=\overline{c^{\prime}(-p,-q)} \\
\Delta^{\prime}(p)=\overline{\Delta^{\prime}(-p)}=-\overline{\Delta^{\prime}(p)} .
\end{gathered}
$$

We note that the invariance under proper Lorentz transformations fixes the parity (positive) under space-inversions:

$$
\begin{gathered}
c^{\prime}\left(p^{0}, \mathbf{p} ; q^{0}, \mathbf{q}\right)=c^{\prime}\left(p^{0},-\mathbf{p} ; q^{0},-\mathbf{q}\right) \\
\Delta^{\prime}(p)=\overline{\Delta^{\prime}(-p)}=-\overline{\Delta^{\prime}(p)} .
\end{gathered}
$$

Proof: Without loss of generality $p$ and $q$ both lie in the $x^{1}, x^{2}$-plane. The transformation $x^{0} \rightarrow x^{0}, x^{1} \rightarrow-x^{1}, x^{2} \rightarrow-x^{2}, x^{3} \rightarrow x^{3}$ is a proper Lorentz transformation.

The invariants $p^{2}, q^{2}$ and $(p+q)^{2}$ determine the vectors $p$ and $q$ up to a proper Lorentz transformation and (possibly) a space-time inversion (or equivalently a time-inversion). Thus

with

$$
c^{\prime}(p, q)=\hat{c}\left(p^{2}, q^{2},(p+q)^{2} ; \varepsilon\right) \quad \text { where } \quad \varepsilon=+ \text { or - }
$$

and

$$
\hat{c}\left(p^{2}, q^{2},(p+q)^{2} ;+\right)=\overline{\hat{c}\left(p^{2}, q^{2},(p+q)^{2} ;-\right)}
$$

$$
\Delta^{\prime}(p)=\varepsilon\left(p^{0}\right) \hat{\Delta}\left(p^{2}\right), \quad \operatorname{Re} \Delta^{\prime}(p)=0 .
$$

Since $\Delta$ is only incidental to the Lie field existence problem (although it may be crucial to the existence of a physically interesting field theory), we shall concern ourselves from now on almost exclusively with the generalized function $c^{\prime}(p, q)$ (or alternatively the Lie bracket operation $[f, g]$ in $\mathscr{K})$.

Time-reversal and charge-conjugation invariance are not implied by the defining axioms of the scalar Lie field, but it is convenient for the sake of algebraic simplicity to require the following sort of TCP invariance (corresponding to negative TCP parity).

For arbitrary $f, g \in \mathscr{K}$,

$$
[f, g]_{0}=\left[f_{\theta}, g_{0}\right], \text { where } f_{0}(x)=f(-x) .
$$

In terms of $c^{\prime}(p, q)$ we have

$$
\begin{aligned}
& c^{\prime}(p, q)=\hat{c}\left(p^{2}, q^{2},(p+q)^{2}\right) \\
& c^{\prime}(p, q)=\overline{c^{\prime}(p, q)} .
\end{aligned}
$$




\section{Some General Results}

In this section we derive some general properties of the scalar Lie field structures satisfying the "TCP invariance" condition (2.4). The arguments are of a formal nature, in that the function space $\mathscr{K}$ of the previous section is not specified. However, the existence of numerous examples (see Section 4 for the simplest of these) for which the statements of this section are applicable indicates that the latter can be made mathematically rigorous if sufficient restrictions are placed on $\mathscr{K}$.

We first consider the Jacobi identity (2.3) (ii). In order to exploit the Lorentz invariance, it is convenient to introduce, in place of the triad $p, q, r$ of four-vectors, the following invariant variables ${ }^{3}$ :

$$
\begin{array}{rlrl}
u & =p^{2} & w & =(p+q)^{2} \\
v & =q^{2} & w^{\prime} & =(q+r)^{2} \\
u^{\prime} & =r^{2} & t & =(r+p)^{2} \\
v^{\prime} & =(p+q+r)^{2} & &
\end{array}
$$

where $u+v+u^{\prime}+v^{\prime}-w-w^{\prime}-t=0$,

and six of the seven variables specify the triad $p, q, r$ up to a not necessarily proper) Lorentz transformation. We further define ${ }^{3}$

$$
C\left(u, v|u| u^{\prime}, v^{\prime}\right) \equiv \hat{c}(u, v, w) \hat{c}\left(w, u^{\prime}, v^{\prime}\right)
$$

so that the Jacobi identity reads

$$
C\left(u, v|w| u^{\prime}, v^{\prime}\right)+C\left(v, u^{\prime}\left|w^{\prime}\right| u, v^{\prime}\right)+C\left(u^{\prime}, u|t| v, v^{\prime}\right)=0 .
$$

It should be kept in mind that equation (3.1) is not valid for all values of the variables $u, v, u^{\prime}, v^{\prime}, w$ and $w^{\prime}$, but rather only for those values which correspond to triads of four-vectors (this is the point overlooked by Robinson).

For fixed $u, v, u^{\prime}$ and $v^{\prime}$ equation (3.1) takes the form

$$
f(w)+g\left(w^{\prime}\right)+h\left(w+w^{\prime}\right)=0,
$$

where $w$ and $w^{\prime}$ vary over a certain region of the $w, w^{\prime}$-plane (see Appendix for details), the geometrically allowed region. Differentiating (3.2), we see that $\frac{\partial}{\partial w} C\left(u, v|w| u^{\prime}, v^{\prime}\right)$ takes on the same value on any two geometrically allowed subregions of the $w, w^{\prime}$-plane which are intersected by a common line $w=$ constant, $w^{\prime}=$ constant or $w+w^{\prime}=$ constant. For certain values of $u, v, u^{\prime}$ and $v^{\prime}$ the allowed region (see Fig. 1) consists of two disjoint subregions, $R_{1}$ and $R_{2}$, such that for all $\left(w_{1}, w_{1}^{\prime}\right)$ in $R_{1}$ and $\left(w_{2}, w_{2}^{\prime}\right)$ in $R_{2}, w_{1} \neq w_{2}, w_{1}^{\prime} \neq w_{2}^{\prime}$ and $w_{1}+w_{1}^{\prime} \neq w_{2}+w_{2}^{\prime}$. For these values of the parameters $\frac{\partial}{\partial w} C\left(u, v|w| u^{\prime}, v^{\prime}\right)$ may take on two values,

\footnotetext{
3 Notation used by Robinson [4].
} 


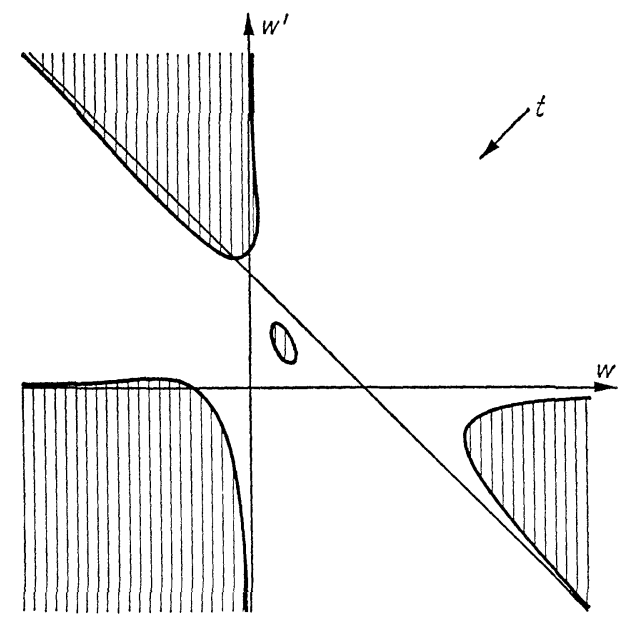

Fig. 1. Sketch of typical geometrically allowed region (shaded) for $v^{\prime}>u^{\prime}>v>$ $>u>0$

$B_{1}\left(u, v, u^{\prime}, v^{\prime}\right)$ and $B_{2}\left(u, v, u^{\prime}, v^{\prime}\right)$; otherwise it is constant over the entire geometrically allowed region and we may write

$$
\frac{\partial}{\partial w} C\left(u, v|w| u^{\prime} v^{\prime}\right)=B_{1}\left(u, v, u^{\prime}, v^{\prime}\right) \text {. }
$$

As observed by RoBInson [4], the product structure

implies that

$$
C\left(u, v|w| u^{\prime}, v^{\prime}\right)=\hat{c}(u, v, w) \hat{c}\left(w, u^{\prime}, v^{\prime}\right)
$$

$$
B_{i}\left(u, v, u^{\prime}, v^{\prime}\right)=G_{i}(u, v) H_{i}\left(u^{\prime}, v^{\prime}\right),
$$

where, on account of the antisymmetry requirement (2.3) (i),

$$
G_{i}(u, v)=-G_{i}(v, u) .
$$

The Jacobi identity (3.1) (differentiated) now implies

$$
G_{i}(u, v) H_{i}\left(u^{\prime}, v^{\prime}\right)=G_{i}\left(v, u^{\prime}\right) H_{i}\left(u, v^{\prime}\right)=G_{i}\left(u^{\prime}, u\right) H_{i}\left(v, v^{\prime}\right) \text {. }
$$

If the lefthand side is nonzero for some choice of $u, v, u^{\prime}$ and $v^{\prime}$, we may fix $u^{\prime}$ and $v^{\prime}$ and divide (3.5) through by $H_{i}\left(u^{\prime}, v^{\prime}\right) \neq 0$ to obtain

$$
G_{i}(u, v)=P_{i}(u) Q_{i}(v) .
$$

But it is easily seen that (3.6) is consistent with (3.4) only if

and hence

$$
G_{i}(u, v)=0
$$

$$
\frac{\partial}{\partial w} C\left(u, v|u| u^{\prime}, v^{\prime}\right)=0
$$


If $\chi_{u v u^{\prime} v^{\prime}}^{i}(w)$ is the characteristic function of the $i$ th allowed interval of the $w$-axis for given $u, v, u^{\prime}$ and $v^{\prime}$ (there are at most three such intervals - one bounded, one unbounded to the left and one unbounded to the right), equation (3.7) allows us to write

$$
C\left(u, v|w| u^{\prime}, v^{\prime}\right)=\sum_{i} \chi_{u v u^{\prime} v^{\prime}}^{i}(w) A_{i}\left(u, v, u^{\prime}, v^{\prime}\right),
$$

where, using the arguments leading to (3.3) and (3.4),

$$
\begin{aligned}
A_{i}\left(u, v, u^{\prime}, v^{\prime}\right) & =E_{i}(u, v) F_{i}\left(u^{\prime}, v^{\prime}\right) \\
E_{\imath}(u, v) & =-E_{i}(v, u) .
\end{aligned}
$$

Thus far we have obtained some general results concerning the form of the product function $\hat{c}(u, v, w) \hat{c}\left(w, u^{\prime}, v^{\prime}\right)$. Now let us use these results and the Jacobi identity

$$
\begin{aligned}
\hat{c}(u, v, w) \hat{c}\left(w, u^{\prime}, v^{\prime}\right)+\hat{c}\left(v, u^{\prime}, w^{\prime}\right) \hat{c}\left(w^{\prime}, u, v^{\prime}\right)+ \\
+\hat{c}\left(u^{\prime}, u, t\right) \hat{c}\left(t, v, v^{\prime}\right)=0
\end{aligned}
$$

to obtain additional information about $\hat{c}(u, v, w)$ itself.

Suppose $\hat{c}(u, v, w) \hat{c}\left(w, u^{\prime}, v^{\prime}\right)$ is nonzero for some choice of variables. Then this quantity remains nonzero (in fact is constant) if we hold $u, v, u^{\prime}$ and $v^{\prime}$ fixed and vary $w$ within the interval $I_{i}$ (notation as in $(3.9))$. Equation (3.10) with $u^{\prime}$ and $v^{\prime}$ fixed implies that $\hat{c}(u, v, w)$ has the form

$$
\hat{c}(u, v, w)=F(w)[A(u) B(v)-C(u) D(v)]
$$

for $w$ in $I_{i}$. Antisymmetry then requires

for $w$ in $I_{i}$.

$$
\hat{c}(u, v, w)=F(w)[A(u) B(v)-B(u) A(v)]
$$

It is clear that the set

$\left\{w\right.$ : there exist $u, v, u^{\prime}, v^{\prime}$ such that $\left.\hat{c}(u, v, w) \hat{c}\left(w, u^{\prime}, v^{\prime}\right) \neq 0\right\}$

is partitioned into a number of intervals on each of which $\hat{c}(u, v, w)$ has the form (3.11). There is no limit on the number of such intervals; of course $\hat{c}(u, v, w) \hat{c}\left(w, u^{\prime}, v^{\prime}\right)$ must vanish on all but (at most) three of them for fixed $u, v, u^{\prime}$ and $v^{\prime}$.

At this point we have arrived at some fairly general conditions on $\hat{c}(u, v, w)$ and $C\left(u, v|w| u^{\prime}, v^{\prime}\right)$. Unfortunately these conditions are not restrictive enough to permit an immediate listing of all possible scalar Lie field structures satisfying the requirements set forth in Section 2. In particular there may be some values of the variable $w$ for which $C\left(u, v|w| u^{\prime}, v^{\prime}\right)=0$ for all $u, v, u^{\prime}$ and $v^{\prime}$ but for which $\hat{c}(u, v, w)$ or $\hat{c}\left(w, u^{\prime}, v^{\prime}\right)$ does not vanish identically. Nevertheless our partial classification of scalar Lie field structures encompasses a wide variety of examples, the simplest of which are presented in the next section. 


\section{Examples}

Example $A$. We designate by $R_{i}, i= \pm 1, \pm 2$, the following regions of Minkowski space:

$$
\begin{aligned}
& R_{ \pm 1}: m_{0}^{2}<p^{2}<m_{1}^{2} \quad p^{0} \gtrless 0 \\
& R_{ \pm 2}: m_{2}^{2}<p^{2}<m_{3}^{2} \quad p^{0} \gtrless 0
\end{aligned}
$$

where $0<m_{0}<m_{1}<m_{2} \ll m_{3}$.

If $\chi_{i}$ is the characteristic function of region $R_{i}$, we define

$$
c^{\prime}(p, q) \equiv\left[\chi_{+2}(p) \chi_{+1}(q)-\chi_{+1}(p) \chi+2(q)\right] \chi_{+2}(p+q)+\left(\begin{array}{l}
+1 \rightarrow-1 \\
+2 \rightarrow-2
\end{array}\right),
$$

or, in terms of the invariant variables $u, v$ and $w$,

$$
\hat{c}(u, v, w) \equiv\left[\chi_{2}(u) \chi_{1}(v)-\chi_{1}(u) \chi_{2}(v)\right] \theta(v-u-v) \chi_{2}(w),
$$

where $\chi_{1}(u)=1$ for $p \in R_{+1} \cup R_{-1}$, etc.

It is worthwhile to point out that in the case $m_{3}=\infty$, there exists a Hilbert space representation of this Lie field structure. Suppose we havea representation of the canonical commutation or anticommutation relations in six dimensions:

$$
\begin{aligned}
{\left[\psi(x, \eta), \psi\left(x^{\prime}, \eta^{\prime}\right)\right]_{ \pm} } & =0 \\
{\left[\psi(x, \eta), \psi^{+}\left(x^{\prime}, \eta^{\prime}\right)\right]_{ \pm} } & =\delta^{4}\left(x-x^{\prime}\right) \delta^{2}\left(\eta-\eta^{\prime}\right)
\end{aligned}
$$

where $x$ is a four-vector and $\eta$ is a two-vector variable. Defining (if meaningful)

$$
\begin{aligned}
\mathscr{T}_{k}(x) & =\frac{1}{2 i} \int d^{2} \eta \psi^{+}(x, \eta) \frac{\overleftrightarrow{\partial}}{\partial \eta_{k}} \psi(x, \eta) \\
\mathscr{D}(x) & =\frac{1}{2 i} \int d^{2} \eta \eta_{k} \psi^{+}(x, \eta) \frac{\overleftrightarrow{\partial}}{\partial \eta_{k}} \psi(x, \eta) \\
\mathscr{R}(x) & =\frac{1}{2 i} \int d^{2} \eta \varepsilon_{i j 3} \eta_{i} \psi^{+}(x, \eta) \frac{\overleftrightarrow{\partial}}{\partial \eta_{i}} \psi(x, \eta)
\end{aligned}
$$

one readily verifies that

$$
A(f) \equiv \mathscr{T}_{1}\left(\operatorname{Re} f_{2}\right)-\mathscr{T}_{2}\left(\operatorname{Im} f_{2}\right)+\mathscr{D}\left(\operatorname{Re} f_{1}\right)-\mathscr{R}\left(\operatorname{Im} f_{1}\right),
$$

where $f_{i}(x)$ is the Fourier transform of $f_{i}(p)=\chi_{i}\left(p^{2}\right) f(p)$, provides a Hermitian representation of the Lie field structure:

where

$$
i[A(f), A(g)]=A([f, g])
$$

$$
[f, g]=2 \operatorname{Re}\left(f_{2} g_{1}-f_{1} g_{2}\right) \text {. }
$$

An example of a representation of the canonical commutation relations in which the $A(f)$ are meaningful and in which there exists a unitary representation of the Poincaré group is the Fock representation. 
Example B. Using the same notation as in Example A, define $c^{\prime}(p, q) \equiv\left[\chi_{+2}(p) \chi_{-1}(q)-\chi_{-1}(p) \chi_{+2}(q)\right] \chi_{+2}(p+q)+\left(\begin{array}{l}-1 \rightarrow+1 \\ +2 \rightarrow-2\end{array}\right)$.

Example $C$. Label space-time regions $R_{i}, i=1, \ldots, 4$, as follows:

$$
\begin{aligned}
& R_{1}: m_{0}{ }^{2}<p^{2}<m_{1}{ }^{2} \\
& R_{2}: m_{2}{ }^{2}<p^{2}<m_{3}{ }^{2} \\
& R_{3}: m_{1}{ }^{2}<p^{2}<m_{2}{ }^{2}
\end{aligned}
$$

where $0<m_{0}<m_{1}<m_{2}<m_{3}$ and $m_{3}-m_{0}<m_{0}$. Define

$$
c^{\prime}(p, q)=\left[\chi_{1}(p) \chi_{2}(q)-\chi_{2}(p) \chi_{1}(q)\right]+\left[\chi_{2}(q)-\chi_{2}(p)\right] \chi_{3}(p+q) .
$$

In each of the above examples one can verify [5] that the given $c^{\prime}(p, q)$ satisfies the requirements of a scalar Lie field structure. The choice of the test-function space $\mathscr{K}$ (see Section 2) is not unique, but in each case one can choose $\mathscr{K}$ to be a space of square-integrable functions whose Fourier transforms decrease faster than any power at infinity.

In all three of the examples we may incorporate into the Lie field structure a $c$-number term defined by a generalized function $\Delta^{\prime}(p)$ (see Section 2), but only in an essentially trivial manner (i.e. the retarded functions calculated from the whole Lie field structure would be essentially those of a generalized free field). For instance, in Example A we could define

$$
\Delta^{\prime}(p)=i\left[\chi_{+1}(p)-\chi_{-1}(p)\right] .
$$

The Jacobi identity then follows, since

$$
c_{1}^{\prime}(p, q) \Delta(-(p+q))=0 .
$$

\section{Euclidean Lie Field Structures}

The existence of scalar, Euclidean-invariant Lie field structures over three-space is a direct corollary of the results of Section 3 concerning Lie field structures over four-dimensional space-time. We recall (changing four-vectors to three-vectors) that the Fourier transform $\widetilde{A}(\mathrm{p})$ of a scalar Lie field satisfies

$$
i[\tilde{A}(\mathbf{p}), \widetilde{A}(\mathbf{q})]=\hat{c}(u, v, w) \widetilde{A}(\mathbf{p}+\mathbf{q}),
$$

where $u=-\mathbf{p}^{2}, v=-\mathbf{q}^{2}$ and $w=-(\mathbf{p}+\mathbf{q})^{2}$, and $\hat{c}(u, v, w)$ is a real generalized function satisfying

$$
\begin{aligned}
& \hat{c}(u, v, w)=-\hat{c}(v, u, w) \\
& \hat{c}(u, v, w) \hat{c}\left(w, u^{\prime}, v^{\prime}\right)+\hat{c}\left(v, u^{\prime}, w^{\prime}\right) \hat{c}\left(w^{\prime}, u, v^{\prime}\right)+\hat{c}(u, v, w) \hat{c}\left(t, v, v^{\prime}\right)=0, \\
& \text { where } u^{\prime}=-\mathbf{r}^{2}, v^{\prime}=-(\mathbf{p}+\mathbf{q}+\mathbf{r})^{2}, t=-(\mathbf{p}+\mathbf{r})^{2}, w^{\prime}=-(\mathbf{q}+\mathbf{r})^{2} .
\end{aligned}
$$


As in Section 3 the possible values of $w, w^{\prime}$ and $t$ for fixed $u, u^{\prime}, v$ and $v^{\prime}$ are restricted by the geometry: the configuration is always such that in the $w, w^{\prime}$-plane the geometrically allowed region is confined to the interior of a closed curve in the bounded region $w<0, w^{\prime}<0, t<0$. The equation of this curve, corresponding to $\mathbf{p}, \mathbf{q}$ and $\mathbf{r}$ all coplanar, is given in the Appendix, equation (A.1).

It is obvious that the scalar Euclidean Lie field structures are in one-one correspondence with a certain subclass of the Lorentz-invariant Lie field structures discussed in Section 3, namely those for which $\hat{c}(u, v, w) \hat{c}\left(w, u^{\prime}, v^{\prime}\right)$ is nonzero only for all arguments positive; for these Lie structures the geometrically allowed region is as shown in Fig. 1 (bounded part of the shaded region only). Examples A and B of Section 4 belong to this class. The crucial point in the construction of these examples is the partition of the timelike region of space-time into forward and backward cones: if $p$ lies along the 0 -axis, timelike $q$ lies in the forward (resp. backward) cone if $p \cdot q>0$ (resp. $p \cdot q<0$ ). Precisely the same situation prevails in the three-dimensional case, with the cones (halfspaces) $\mathbf{p} \cdot \boldsymbol{q}>0$ and $\mathbf{p} \cdot \boldsymbol{q}<0$. The Euclidean analogs of these examples may be written down immediately:

Example $\mathrm{A}^{\prime}$ : where

$$
\hat{c}(u, v, w)=\left[\chi_{2}(u) \chi_{1}(v)-\chi_{1}(u) \chi_{2}(v)\right] \theta(u+v-w) \chi_{2}(v),
$$

$$
\begin{aligned}
& \chi_{1}(u)=\left\{\begin{array}{ll}
1 & k_{0}{ }^{2}<-u<k_{1}{ }^{2} \\
0 & \text { otherwise }
\end{array} \quad k_{2}^{2}>k_{1}^{2}\right. \\
& \chi_{2}(u)= \begin{cases}1 & k_{2}{ }^{2}<-u<k_{3}^{2} \\
0 & \text { otherwise. }\end{cases}
\end{aligned}
$$

Example $B^{\prime}$ :

$$
\hat{c}(u, v, w)=\left[\chi_{2}(u) \chi_{1}(v)-\chi_{1}(u) \chi_{2}(v)\right] \theta(w-u-v) \chi_{2}(w)
$$

(same notation as in Example $A^{\prime}$ ).

\section{Appendix. Geometrically Allowed Region}

In this appendix we describe in detail the geometrically allowed regions of the $w, w^{\prime}$-plane for a given set of parameters $u, v, u^{\prime}$ and $v^{\prime}$.

It is useful to observe that throughout the discussion of antisymmetry and the Jacobi identity we always deal with a sub-space of spacetime of three or fewer dimensions spanned by vectors $p, q$, and $r$. We may consider separately the three cases characterized by whether the vector orthogonal to the $p, q, r$-triad is (a) spacelike, (b) timelike, or (c) lightlike. In each case the range of $w^{\prime}$ for fixed $u, v, u^{\prime}, v^{\prime}$ and $w$ can be ascertained by straightforward geometrical arguments. 
(a) $p, q$, and $r$ orthogonal to a spacelike vector. Define $p \times q$ normal to the $p, q$-plane by

$$
(p \times q)_{\mu}=\varepsilon_{\mu \nu \varrho} p^{v} q^{\varrho} \quad \text { with } \quad(p \times q)^{2}=p^{2} q^{2}-(p \cdot q)^{2} .
$$

The indices run from 0 to 2 and the signature of the metric is + - -

(i) $w>0, \quad(p \times q)^{2}((p+q) \times r)^{2}>0$ :

$w^{\prime}$ lies between the coplanar extremes (those values achieved when $p, q$, and $r$ are all coplanar).

(ii) $w>0, \quad(p \times q)^{2}((p+q) \times r)^{2}<0$ :

Geometrically impossible.

(iii) $w>0, \quad(p \times q)^{2}((p+q) \times r)^{2}=0$ :

Borderline case: only one value of $w^{\prime}$ is possible.

(iv) $w<0, \quad(p \times q)^{2}((p+q) \times r)^{2}>0$ :

$w^{\prime}$ lies outside the coplanar extremes; $w^{\prime}$ is not bounded.

(v) $w<0, \quad(p \times q)^{2}((p+q) \times r)^{2} \leqq 0$ :

(vi) $w=0$ :

$$
-\infty<w^{\prime}<+\infty \text {. }
$$

$w^{\prime}$ may take on any value on a half-line with the extremal value corresponding to $p, q$, and $r$ coplanar.

(b) $p, q$, and $r$ orthogonal to a timelike vector. This is the case of Euclidean 3-space. As in (a) (i) $w^{\prime}$ lies between the coplanar extremes.

(c) $p, q$, and $r$ orthogonal to a lightlike vector. This is the case of Euclidean 2 -space and $w^{\prime}$ takes on precisely the values achieved when $p, q$ and $r$ are coplanar.

From the foregoing we see that the entire allowed region in the $w$, $w^{\prime}$-plane for fixed $u, v, u^{\prime}$ and $v^{\prime}$ is determined once we know the value of $w^{\prime}$ for $p, q$ and $r$ all coplanar. A straightforward calculation gives

$$
\begin{aligned}
w^{\prime}=v+u^{\prime} & +\frac{1}{2 w}(w-u+v)\left(v^{\prime}-w-u^{\prime}\right) \pm \\
& \pm \frac{1}{2 w} \sqrt{ }\left[(w+v-u)^{2}-4 w v\right]\left[\left(w+u^{\prime}-v^{\prime}\right)^{2}-4 w u^{\prime}\right]
\end{aligned}
$$

or, in geometrically more transparent form,

$$
\begin{aligned}
(q+r)^{2} & =q^{2}+r^{2}+2 \frac{[q \cdot(p+q)][r \cdot(p+q)]}{(p+q)^{2}} \pm \\
\pm & \frac{2}{(p+q)^{2}} \sqrt{\left[(p+q)^{2} q^{2}-((p+q) \cdot q)^{2}\right]\left[(p+q)^{2} r^{2}-((p+q) \cdot r)^{2}\right]} .
\end{aligned}
$$

The third term on the righthand side is, up to sign, twice the product of the components of $q$ and $r$ parallel to $p+q$, and the last term is, again up to sign, twice the product of the components orthogonal to $p+q$. 
The salient features of (A.1) are the following:

(1) Triangular symmetry. The variables $w, w^{\prime}$ and $t=u+v+u^{\prime}+$ $+v^{\prime}-w-w^{\prime}$ are double-valued functions of one another with the same form (A.1) if the parameters $u, v, u^{\prime}$, and $v^{\prime}$ are permuted appropriately. Thus any general properties of the curve (A.1) with respect to the $w, w^{\prime}$-axis must be true with respect to the $w, t$-and $w^{\prime}, t$-axes as well.

(2) Behavior as $w \rightarrow 0$.

$$
w^{\prime} \sim\left\{\begin{array}{l}
\frac{1}{w}(v-u)\left(v^{\prime}-u^{\prime}\right)+\text { constant } \\
\frac{1}{2}\left(u+v+u^{\prime}+v^{\prime}\right)+\frac{1}{2}\left[\frac{\left(u^{\prime}-v^{\prime}\right)(u+v)}{(u-v)}+\frac{(u-v)\left(u^{\prime}+v^{\prime}\right)}{\left(u^{\prime}-v^{\prime}\right)}\right] .
\end{array}\right.
$$

(3) Behavior as $w \rightarrow \infty$.

$$
w^{\prime} \sim\left\{\begin{array}{l}
\frac{1}{w}\left(v-u^{\prime}\right)\left(v^{\prime}-u\right) \\
\left(u+v+u^{\prime}+v^{\prime}\right)-w+\frac{1}{w}\left(u^{\prime}-u\right)\left(v^{\prime}-v\right) .
\end{array}\right.
$$

(4) Critical points. The function (A.1) is single-valued at points where the quantity within the square-root sign vanishes. Negative values of the latter correspond to those values of $w$ which are not attainable geometrically (in two dimensions, at any rate).

Note that (3) actually follows from (1) and (2).

Given any set of parameters $u, v, u^{\prime}$, and $v^{\prime}$ one can use (1)-(4) to obtain a sketch of the curve $w^{\prime}=w^{\prime}(w)$ showing all the important features.

\section{References}

1. Greenberg, O. W.: Ann. Phys. 16, 158 (1961).

2. Eopuszański, J. T.: Phys. Letters 8, 85 (1964).

3. Wightman, A. S.: Lecture notes, Introduction to some aspects of the relativistic dynamics of quantized fields, Cargese, 1964.

4. Robinson, D. W.: Phys. Letters 9, 189 (1964).

5. Lowenstein, J. H.: Ph. D. Thesis, University of Illinois, 1966. 